\title{
Using the Callback to eliminate sub-routing in a vehicle routing problem with simultaneous pick-up and delivery of multiple products
}

Roza Maria Zoellner Lopes ${ }^{1}$, Universidade Federal do Paraná Ruany Batista Leite Doehnert ${ }^{2}$, Universidade Federal do Paraná Gustavo Valentim Loch ${ }^{3}$, Universidade Federal do Paraná José Eduardo Pécora Junior ${ }^{4}$, Universidade Federal do Paraná Cassius Tadeu Scarpin ${ }^{5}$, Universidade Federal do Paraná

\section{ABSTRACT}

The Vehicle Routing Problem with simultaneous pick-up and delivery of multiple products (VRPSPD) is addressed in practice when just collecting the product is not sufficient to meet the client's requirements. This article aims to apply Callback available in Gurobi software to the elimination of sub-routes in a VRPSPD using lazy constraints. In order to test the efficiency of the proposed method, we first solved the relaxed problem of Mixed Integer Programming (MIP), and then the same problem was solved using the Callback. The performed tests show that the use of Callback is efficient leading to time reductions in runtime to prove the optimality.
Editor Responsável: Prof. Dr. Hermes Moretti Ribeiro da Silva

\section{Keywords: Vehicle Routing Problem; Simultaneous Delivery and Pick-Up; Multiple products; Callback; Lazy Constraints}

\footnotetext{
1.Rua XV de Novembro, 1299 - Centro, Curitiba - PR, 80060-000, rozazoellner@gmail.com; 2. ruany.leite@gmail.com; 3. gustavo.gvalentim@gmail.com; 4. pecorajr@gmail.com; 5. cassiusts@gmail.com.

LOPES, R.M.Z.; DOEHNERT, R.B.L.; LOCH, G.V.; PÉCORA JUNIOR, J.E.; SCARPIN, C.T. Using the Callback to eliminate sub-routing in a vehicle routing problem with simultaneous pick-up and delivery of multiple products. GEPROS. Gestão da Produção, Operações e Sistemas, v. 15, n. 3, p. 58 - 68, 2020.

DOI: $10.15675 /$ gepros.v15i3.2726
} 


\section{INTRODUCTION}

The freight transportation is a relevant process for the logistics of the supply chain management, where companies are focusing their efforts to improve the level of service and also to reduce their cost (YOUSEFIKHOSHBAKHT; DIDEHVAR; RAHMATI, 2015).

The vehicle routing with simultaneous pick-ups and deliveries is vital problem to the supply-chain management because it addresses business models where companies should deliver their products and pick-up some returning goods or packings, as in the reverse logistics. Examples of its use are in pallet delivery services, where it is necessary to pick it up later; returnable bottles; retail industry, where products with defects can be picked up, among others (ALSHAMRANI; MATHUR; BALLOU, 2007).

In this article, the problem addressed is based on Zhang et al. (2019), which presents the VRPSPD for a fast-fashion company. Vehicles with homogeneous load leave the warehouse going through sale points only once and return to the warehouse at the end of the route. The products removed from a sale point can be redistributed to meet the demand of the customers of another sale point, making the problem more complex to be solved.

In the computer programming area, there is a widely used resource, the Callback, which allows a return to the code when a specific event occurs (WANG et al., 2018). You can call the Callback function present in some MIP (Mixed Integer Programming) solvers and add lazy constraints. These are restrictions that are not part of the initial problem model but are complementary to it to speed up the problem resolution, being added after a specific condition occurs (LUNDELL; KRONQVIST, 2019).

Therefore, the purpose of this article is to use the Callback resource, in Gurobi ${ }^{\circledR}$ software, to add the restriction that eliminates sub-routes to the VRPSPD problem only when they are previously violated in the incumbent optimal solution. The advantage of this approach relies on the fact that the number of this set of constraints is enormous, as seen in later in expression (6). This procedure aims at decreasing the computational time for solving the exact problem.

The remainder of this article is structured as follows: Section 2 presents the research related to the subject of this article; Section 3 depicts the detailed description of the problem, 
the MIP, a relaxed MIP for the problem and the necessary changes to the implementation of the Callback; Section 4 presents the computational results and Section 5 concludes the paper.

\section{LITERATURE REVIEW}

This section presents a review of the literature of similar works to the base article of Zhang et al. (2019) to identify the solving approaches used.

Alshamrani et al. (2007) addressed the problem of blood distribution in hospitals. Blood is delivered in thermal boxes suitable for preservation, and these boxes are reusable. Therefore, the problem consists of simultaneous delivery of the blood and pick-up the empty boxes with a single vehicle with limited capacity. If, when delivering the blood, the vehicle does not have enough capacity to collect empty boxes, the collection may be postponed at a penalty. The objective function minimizes the route and the penalty for not being able to remove the empty boxes on the same delivery trip. A two-part solution is performed: first, the solution for the routing is found using an arbitrary insertion procedure; second, the weighted leveling heuristic is used to consider the penalty costs involved.

Wang (2018) tackled the problem of delivering meals to several suppliers. The logistics service collects the products from the suppliers and delivers them to the customers, within a maximum time for delivery. The vehicles may leave from different deposits. The methodology used was to compare the implementation of three logistics services: Exclusive where each vehicle serves only one supplier; Shared - one vehicle can serve several suppliers in sequence; and Shared - each vehicle can serve more than one supplier while still on the route of the previous one. The problem was solved using two heuristics: Iterated Local Search (ILS) and Adaptive Large Neighborhood Search (ALNS). The used heuristics obtained good results for big problems, however it was not possible to find the optimal solution.

Bouanane, Benadada and Bencheikh (2018) modeled and solved the problem to deliver and pick up products for several customers with multiple warehouses, considering the stock level. The objective of the study was to minimize the cost of traveling and vehicle usage. The problem was solved by developing a hybrid genetic algorithm (GA).

Li et al. (2018) address the VRP with the use of Callback. The study consists of determining the route of tractors carrying loaded containers to customers and returning with empty ones within a time window. The problem aims to minimize the total operational cost of 
tractors. The proposed MILP problem is solved by a Benders decomposition algorithm with cuts for small instances. The Callback is used to define the cuts that must be performed. Larger problems are solved by a local search heuristic.

Guimarães et al. (2019) solved the two Echelons Multi Depot Inventory Routing Problem (2E-MDIRP). This problem consists of a vendor managed inventory considering two echelons in the supply chain. It is considered the replenishment of materials from intermediate customers as well as the final customer. They solve the 2E-MDIRP with an exact model, and a hybrid technique that combines local search with the exact model was used.

Hof and Schneider (2019) solved the VRPSPD and two other variants: the first imposing a time limit for the routes and the second taking into account time windows for the delivery. They solved these problems with a hybrid heuristic, containing an Adaptive Large Neighborhood Search Algorithm with Path Relinking Approach. The results obtained were compared with benchmark instances obtained in the literature.

Golsefidi and Jokar (2020) presented the MILP for the VRPSPD and solved with a Genetic Algorithm (GA) and a Simulated Annealing (SA). The objective of the study was to obtain a reduction in product delivery and collection of defective product costs.

Hornstra et al. (2020) solved the VRPSPD minimizing the costs of product collection, delivery, and handling. They solved it with benchmark and self-generated instances using the exact MIP and an ALNS.

All articles in this section solved the VRPSPD using a constraint in its initial mathematical model to avoid the formation of subroutines. Our proposal is to generate such constraints in running time using Callbacks.

\section{METHODOLOGICAL PROCEDURES}

This article solves the same problem as in Zhang et al. (2019) in the fast-fashion industry. An unlimited and homogeneous fleet delivers products to stores and pick-up those items that are not being sold using the same route. The items removed from one store can be redistributed to others, increasing the complexity of the problem, since the replenishment items do not necessarily have to leave the warehouse.

Vehicles must return to the warehouse at the end of the route, and each customer can be visited only once. The costs of transporting the products from one sale point to another and 
Using the Callback to eliminate sub-routing in a vehicle routing problem with simultaneous pick-up and delivery of multiple products

the costs of transporting the products from the warehouse are considered. In Zhang et al. (2019), the distance matrix is asymmetric.

\subsection{Mathematical model}

The indices, parameters, variables, objective function, and constraints from Zhang et al. (2019) from both the exact model and a relaxed model are presented below.

\subsubsection{Mixed Integer Programming to VRPSPD}

Source: The authors.

\begin{tabular}{c|l}
\multicolumn{2}{c}{ Table $\mathbf{1}$ - Indexes of the model } \\
\hline$i$ & Origin node \\
$j$ & Destination node \\
$h$ & Product \\
\hline
\end{tabular}

Table 2 - Parameters of the model

\begin{tabular}{|c|c|}
\hline \multicolumn{2}{|r|}{ Parameters } \\
\hline$n$ & Number of sales points \\
\hline$m$ & Number of different products \\
\hline$V$ & Set of the depot and the sales points $(V=\{0,1, \ldots, n\})$ \\
\hline$S$ & Set of the sales points $(S=\{1,2, \ldots, n\})$ \\
\hline$H$ & Set of the different products $(H=\{1,2, \ldots, m\})$ \\
\hline$C_{i j}$ & Travel cost from node $i$ to $j$ \\
\hline$w$ & Cost of collect/delivery products on the depot \\
\hline$-d_{i h}$ & Amount of product $h$ delivered to node $i$, if $d i h<0$ \\
\hline$d_{i h}$ & Amount of product $h$ collected from node $i$, if $d i h>0$ \\
\hline Cap & Capacity of each vehicle \\
\hline
\end{tabular}

Source: The authors. 
Using the Callback to eliminate sub-routing in a vehicle routing problem with simultaneous pick-up and delivery of multiple products

Table 3 - Decision variables of the model

\begin{tabular}{c|l}
\hline \multicolumn{1}{c}{ Decision variables } \\
\hline$X_{i j}$ & Binary variable, 1 if vehicle traverses arc $(i, j) ; 0$ otherwise \\
$Q_{i j h}$ & Amount of product $h$ delivered on $\operatorname{arc}(i, j)$ \\
$B_{i}$ & Auxiliary variables to eliminate sub-tour \\
\hline
\end{tabular}

Source: The authors.

Mixed Integer Programming Model

$\operatorname{Min} \sum_{i \in V} \sum_{j \in V} C_{i j} X_{i j}+\sum_{i \in S} \sum_{h \in H} w\left(Q_{0 i h}+Q_{i 0 h}\right)$

$\mathrm{s} / \mathrm{a}$

$\sum_{j \in V \backslash\{i\}} X_{i j}=1, \quad \forall i \in S$

$\sum_{j \in V \backslash\{i\}} X_{j i}=1, \quad \forall i \in S$

$\sum_{j \in V \backslash\{i\}} Q_{j i h}+d_{i h}=\sum_{j \in V \backslash\{i\}} Q_{i j h}, \quad \forall i \in S, h \in H \neq i$

$0 \leq \sum_{h=H} Q_{i j h} \leq \operatorname{CapX}_{i j,} \quad \forall i, j \in V_{j} j \neq i$

$B_{j} \geq B_{i}+1-\left(1-X_{i j}\right) M, \quad \forall i, j \in S_{j} j \neq i$

$X_{i j} \in\{0,1\}, \forall i, j \in V$

$Q_{i j h} \in Z^{+}, \quad \forall i, j \in V_{j} j \neq i, h \in H$

$B_{i} \geq 0, \quad \forall i \in S$

GEPROS. Gestão da Produção, Operações e Sistemas, v. 15, nº 3, p. 58 - 68, 2020. 
Using the Callback to eliminate sub-routing in a vehicle routing problem with simultaneous pick-up and delivery of multiple products

The objective function (1) minimizes the delivery costs. Constraint sets (2) and (3) guarantee that each node is visited exactly once. Constraint set (4) ensures that each demand is collected and delivered. Constraint set (5) imposes that vehicle capacity is not exceeded. Constraint set (6) eliminate sub-tours. Moreover, $M$ may be set equals to $n$. Constraint sets (7)- (9) establish the domain of each decision variable.

\subsubsection{Relaxed Model}

In the model (1)-(9) the integrality constraints of variables $Q_{i j h}$ are eliminated by adding the constraints (10)-(13), i. e., the constraint (8) may be replaced by constraints (10)(13), leading to a relaxed version of the model (1)-(9).

$$
\begin{aligned}
& d_{i h} \leq \sum_{j \in V \backslash\{i\}} Q_{i j h} \leq \operatorname{Cap}+d_{i h}, \quad \forall i \in S, h \in H \\
& -d_{i h} X_{i j} \leq Q_{i j h} \leq \operatorname{Cap}-d_{i h} X_{i j}, \quad \forall i, j \in S, j \neq i, h \in H \\
& \sum_{j \in V \backslash\{i\}} \sum_{h \in H} Q_{i j h} \leq \operatorname{Cap}+\sum_{h \in H} d_{i h}, \quad \forall i \in S \\
& \sum_{h=H} Q_{i j h} \leq \operatorname{Cap}-X_{i j} \sum_{h=H} d_{j h}, \forall i, j \in S, j \neq i
\end{aligned}
$$

\subsubsection{CallBack}

The Callback resource was used to dynamically add the constraints of (6) only when they are violated. It is important to note that the number of constraints in this set is high, and its inclusion a priori on the model may not be practicable. Therefore, we solve the model 
Using the Callback to eliminate sub-routing in a vehicle routing problem with simultaneous pick-up and delivery of multiple products

without considering this set constraint, and once an optimal solution is found, we check if any of this constraint is violated, i. e., if there exist sub-tours in the solution. If a sub-tour is found, then the constraint to prohibit this sub-tour is added as a lazy constraint.

Thus, in the relaxed MIP model the constraint set (6) is removed. Morevover, we add constraints to eliminate the trivial sub-tour $i-i-i$ by setting $\boldsymbol{x}_{i i}=\mathbf{0}, \forall \boldsymbol{i}$.

When the call-back is called we check if the incumbente solution is optimal to the current constraints. If in an optimal solution we have tours of length smaller than $n$ it means we have a soubtour and the constraint (14) is added as Lazy and the problems resumes to be solved once the incumbent solution is not optimal to the new set of constraints.

$$
\sum_{i=0}^{n t} \sum_{j=0}^{n t} X_{i j} \leq \text { Tour }-1 \quad \forall X_{i j} \in \text { Tour }
$$

\section{RESULTS AND DISCUSSION}

The computational experiments to compare the results of the different models were conducted in a computer intel core i5-8250U, with 8Gb of RAM, and the Gurobi 8.1.1 was the solver used. Moreover, the time limit was set to two hours.

In order to validate the model and provide an initial comparison, we used two small instances for both the relaxed model proposed by Zhang et al. (2019) without and with the usage of Callback. The results are presented in Table 4.

Table 4 - initial results

\begin{tabular}{cccccccccc} 
& & & & \multicolumn{4}{c}{ Without Callback } & \multicolumn{3}{c}{ With Callback } \\
\hline instance & $\mathbf{n}$ & $\mathbf{m}$ & cap & OF & Gap & Time (s) & OF & Gap & time(s) \\
\hline 1 & 2 & 2 & 15 & 698,6 & $0,00 \%$ & 0,01 & 698,6 & $0,00 \%$ & 0,01 \\
2 & 3 & 2 & 15 & 1210 & $0,00 \%$ & 0,14 & 1210 & $0,00 \%$ & 0,04 \\
\hline
\end{tabular}

Source: The authors.

The optimal tours for these two instances are shown in Figure 1. For the first one, the optimal route was 0-2-1-0, and for the second one, it was 0-3-1-2-0. 
Using the Callback to eliminate sub-routing in a vehicle routing problem with simultaneous pick-up and delivery of multiple products

Figure 1- Optimal tours for instances 1 and 2, respectively in (a) and (b)
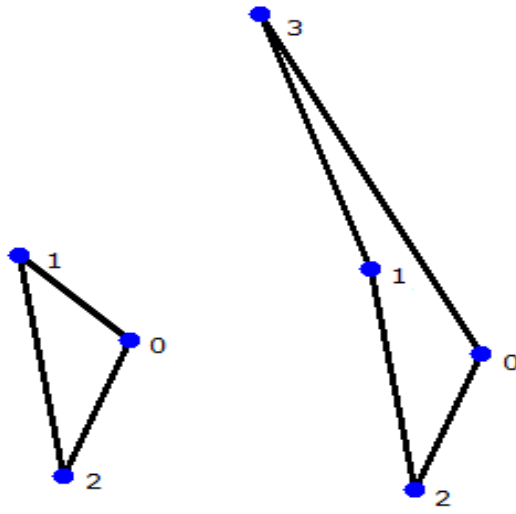

Source: The authors.

Once the model and the usage of callback were validated, we conducted more computational experiments on random generated instances and solved them both without and with the usage of callback. In order to generate the random instances we considered sales points ranging from 10 to 70 , quantity of products from 2 to 4 and vehicles with capacity from 100 to 200 .

The results obtained are presented in Table 5. A trace is used for the cases that no feasible solution was found within the time limit of two hours, and the bold highlights the best runtime for each instance.

Table 5- Computational results

\begin{tabular}{cccccccccc} 
& & & \multicolumn{4}{c}{ Without Callback } & \multicolumn{3}{c}{ With Callback } \\
\hline Instância & $\mathrm{n}$ & $\mathrm{m}$ & Cap & OF & gap & time(s) & OF & gap & time(s) \\
\hline 1 & 10 & 4 & 100 & 2950 & $0,00 \%$ & $\mathbf{0 , 3 1}$ & 2950 & $0,00 \%$ & 0,45 \\
2 & 10 & 4 & 100 & 3006 & $0.00 \%$ & $\mathbf{2 5 , 7 8}$ & 3006 & $0.00 \%$ & 33,09 \\
3 & 20 & 4 & 100 & 4610 & $0,03 \%$ & 5808,99 & 4610 & $0,00 \%$ & $\mathbf{1 3 0 , 7 5}$ \\
4 & 20 & 4 & 100 & 4160 & $7,00 \%$ & $\mathbf{3 9 6 5 . 9 7}$ & - & - & - \\
5 & 30 & 4 & 100 & 4700 & $0,00 \%$ & $\mathbf{1 6 9 2 , 9 9}$ & 4700 & $0,00 \%$ & 2535,92 \\
6 & 30 & 4 & 100 & 8920 & $0.00 \%$ & 43,99 & 8920 & $0.00 \%$ & $\mathbf{2 7 , 0 1}$ \\
7 & 10 & 2 & 200 & 1670 & $0,00 \%$ & 0,18 & 1670 & $0,00 \%$ & $\mathbf{0 , 1 2}$ \\
8 & 10 & 2 & 200 & 1600 & $0,00 \%$ & $\mathbf{0 , 5 3}$ & 1600 & $0,00 \%$ & 0,66 \\
9 & 20 & 2 & 200 & 2110 & $0,00 \%$ & 0,95 & 2110 & $0,00 \%$ & $\mathbf{0 , 6 9}$ \\
10 & 20 & 2 & 200 & 2700 & $0,00 \%$ & $\mathbf{5 , 9 1}$ & 2700 & $0,00 \%$ & 7,53 \\
11 & 30 & 2 & 200 & 2730 & $0,00 \%$ & 10,56 & 2730 & $0,00 \%$ & $\mathbf{5 , 9 9}$ \\
12 & 30 & 2 & 200 & 5080 & $0,00 \%$ & 17,79 & 5080 & $0,00 \%$ & $\mathbf{7 , 8 8}$ \\
13 & 40 & 2 & 200 & 3310 & $0,00 \%$ & 46,4 & 3310 & $0,00 \%$ & $\mathbf{1 5 , 0 3}$
\end{tabular}


Using the Callback to eliminate sub-routing in a vehicle routing problem with simultaneous pick-up and delivery of multiple products

\begin{tabular}{cccccccccc}
14 & 50 & 2 & 200 & 6830 & $0,01 \%$ & 245,68 & 6830 & $0,00 \%$ & $\mathbf{9 8 , 4 4}$ \\
15 & 60 & 2 & 200 & - & - & - & 5120 & $0,01 \%$ & $\mathbf{1 3 3 1 , 8 9}$ \\
16 & 70 & 2 & 200 & - & - & - & - & - & - \\
17 & 10 & 4 & 200 & 2860 & $0,00 \%$ & 0,39 & 2860 & $0,00 \%$ & $\mathbf{0 , 1 4}$ \\
18 & 10 & 4 & 200 & 2750 & $0,00 \%$ & 0,43 & 2750 & $0,00 \%$ & $\mathbf{0 , 2 1}$ \\
19 & 20 & 4 & 200 & 4340 & $0,00 \%$ & 4 & 4340 & $0,00 \%$ & $\mathbf{3}$ \\
20 & 20 & 4 & 200 & 3890 & $0,00 \%$ & 17,74 & 3890 & $0,00 \%$ & $\mathbf{5 , 7 8}$ \\
21 & 30 & 4 & 200 & 4400 & $0,00 \%$ & 44,81 & 4400 & $0,00 \%$ & $\mathbf{2 5 , 6}$ \\
22 & 30 & 4 & 200 & - & - & - & 8570 & $0,00 \%$ & $\mathbf{5 9 9 , 6}$ \\
23 & 40 & 4 & 200 & - & - & - & 5390 & $0,00 \%$ & $\mathbf{8 6 6 , 5 1}$ \\
24 & 40 & 4 & 200 & - & - & - & 1150 & $0,01 \%$ & $\mathbf{1 3 8 9 , 2 7}$ \\
25 & 40 & 4 & 200 & - & - & - & - & - & - \\
\hline
\end{tabular}

Source: The authors.

We have that the time required to find the optimal solution was better with the usage of Callback without using in 17 of the 23 instances that were possible to be solved in less than the time limit. Moreover, the usage advantages of Callback were more significant for larger instances.

\section{CONCLUSIONS}

In this paper, it was proposed and evaluated the usage of callback resources in the solver Gurobi ${ }^{\circledR}$ to add subtour constraints, only when they are violated, as lazy constraints in the MIP VRPSPD proposed by Zhang et al. (2019). Results showed that our proposal led to runtime reductions up to $74 \%$ over randomly generated instances.

The same strategy of using lazy constraints may be easily adapted to other MIP problems with constraint sets that the number of constraints is large, but when the problem is solved without them only few are violated. Moreover, as this strategy is accessible to be implemented in the state-of-the-art Gurobi solver, there is a grate opportunity to solve many real problems that were impossible until some years ago. 
Using the Callback to eliminate sub-routing in a vehicle routing problem with simultaneous pick-up and delivery of multiple products

\section{References}

ALSHAMRANI, A.; MATHUR, K.; BALLOU, R. H. Reverse logistics: simultaneous design of delivery routes and returns strategies. Computers and Operations Research, v. 34, n. 2, p. 595-619, 2007.

BOUANANE, K.; BENADADA, Y.; BENCHEIKH, G. Application of a hybrid genetic algorithm to solve the multi-depots vehicle routing problem with simultaneous delivery and pickup and inventory restrictions. Proceedings - GOL 2018: 4th IEEE International Conference on Logistics Operations Management, n. 4, p. 1-9, 2018.

GUIMARÃES, T. A. et al. The two-echelon multi-depot inventory-routing problem. Computers and Operations Research, v. 101, p. 220-233, 2019.

HEMMATI GOLSEFIDI, A.; AKBARI JOKAR, M. R. A robust optimization approach for the production-inventory-routing problem with simultaneous pickup and delivery. Computers and Industrial Engineering, v. 143, p. 106388, 2020.

HOF, J.; SCHNEIDER, M. An adaptive large neighborhood search with path relinking for a class of vehicle-routing problems with simultaneous pickup and delivery. Networks, v. 74, n. 3, p. 207-250, 2019.

HORNSTRA, R. P. et al. The vehicle routing problem with simultaneous pickup and delivery and handling costs. Computers and Operations Research, v. 115, p. 104858, 2020.

LI, H. et al. The generalized rollon-rolloff vehicle routing problem and savings-based algorithm. Transportation Research Part B: Methodological, v. 113, p. 1-23, 2018.

LUNDELL, A.; KRONQVIST, J. Integration of polyhedral outer approximation algorithms with MIP solvers through callbacks and lazy constraints. AIP Conference Proceedings, v. 2070, n.2, 2019.

WANG, F. et al. Backpropagation with continuation callbacks: Foundations for efficient and expressive differentiable programming. Advances in Neural Information Processing Systems, v. 2018, n. Nips, p. 10180-10191, 2018.

WANG, Z. Delivering meals for multiple suppliers: Exclusive or sharing logistics service. Transportation Research Part E. Logistics and Transportation Review, v. 118, n.8 p. 496$512,2018$.

YOUSEFIKHOSHBAKHT, M.; DIDEHVAR, F.; RAHMATI, F. A Mixed Integer Programming Formulation for the Heterogeneous Fixed Fleet Open Vehicle Routing Problem. Journal of Optimization in Industrial Engineering, v. 18, p. 37-46, 2015.

ZHANG, Z. et al. Multi-commodity demand fulfillment via simultaneous pickup and delivery for a fast fashion retailer. Computers and Operations Research, v. 103, p. 81-96, 2019. 